\title{
Remodularization Analysis for Microservice Discovery Using Syntactic and Semantic Clustering
}

\author{
Adambarage Anuruddha Chathuranga De Alwis ${ }^{1(凶)}(\mathbb{0})$, Alistair Barros ${ }^{1}(\mathbb{D}$, \\ Colin Fidge ${ }^{1}\left(\mathbb{D}\right.$, and Artem Polyvyanyy ${ }^{2}$ (D) \\ 1 Queensland University of Technology, Brisbane, Australia \\ \{adambarage.dealwis, alistair.barros, c.fidge\}@qut.edu.au \\ 2 The University of Melbourne, Parkville, VIC 3010, Australia \\ artem.polyvyanyy@unimelb.edu.au
}

\begin{abstract}
This paper addresses the challenge of automated remodularization of large systems as microservices. It focuses on the analysis of enterprise systems, which are widely used in corporate sectors and are notoriously large, monolithic and challenging to manually decouple because they manage asynchronous, user-driven business processes and business objects (BOs) having complex structural relationships. The technique presented leverages semantic knowledge of enterprise systems, i.e., BO structure, together with syntactic knowledge of the code, i.e., classes and interactions as part of static profiling and clustering. On a semantic level, BOs derived from databases form the basis for prospective clustering of classes as modules, while on a syntactic level, structural and interaction details of classes provide further insights for module dependencies and clustering, based on K-Means clustering and optimization. Our integrated techniques are validated using two open source enterprise customer relationship management systems, SugarCRM and ChurchCRM. The results demonstrate improved feasibility of remodularizing enterprise systems (inclusive of coded BOs and classes) as microservices. Furthermore, the recommended microservices, integrated with 'backend' enterprise systems, demonstrate improvements in key non-functional characteristics, namely high execution efficiency, scalability and availability.
\end{abstract}

Keywords: Microservice discovery $\cdot$ System remodularization $\cdot$ Cloud migration

\section{Introduction}

Microservice architecture (MSA) has emerged as an evolution of service-oriented architecture (SOA) to enable effective execution of software applications in Cloud, Internet-of-Things and other distributed platforms [1]. Microservices (MSs) are fine-grained, in comparison to classical SOA components. They 
entail low coupling (inter-module dependency) and highly cohesive (intra-module dependency) functionality, down to individualised operations, e.g., single operation video-download as a MS component, versus a multi-operation video management SOA component [2]. This promotes systems performance properties, such as high processing efficiency, scalability and availability.

Reported experiences on MS development concern "greenfield" developments [1], where MSs are developed from "scratch". However, major uncertainty exists as to how MSs can be created by decoupling and reusing parts of a larger system, through refactoring. This is of critical importance for the corporate sectors which rely on large-scale enterprise systems (ESs), (e.g., Enterprise Resource Planning (ERP) and Customer-Relationship Management (CRM)), to manage their operations. Analysing ESs and identifying suitable parts for decoupling is technically cumbersome, given the millions of lines of code, thousands of database tables and extensive functional dependencies of their implementations. In particular, ESs manage business objects (BOs) [3], which have complex relationships and support highly asynchronous and typically user-driven processes [4-6]. For example, an order-to-cash process in SAP ERP has multiple sales orders, having deliveries shared across different customers, with shared containers in transportation carriers, and with multiple invoices and payments, which could be processed before or after delivery [7]. This poses challenges to identify suitable and efficient MSs from ES codes using classical software refactoring and optimal splitting/merging of code across software modules.

Software remodularization techniques [8-10] have been proposed based on static analysis, to identify key characteristics and dependencies of modules, and abstract these using graph formalisms. New modules are recommended using clustering algorithms and coupling and cohesion metrics. The focus of static analysis techniques includes inter-module structure (class inheritance hierarchies), i.e., structural inheritance relationships, and inter-module interactions (class object references), i.e., structural interaction relationships. Given that a degradation of logical design reflected in software implementations can result in classes with low cohesion, other techniques have been proposed to compare structural properties of classes using information retrieval techniques [10], i.e., structural class similarity. Despite these proposals, studies show that the success rate of software remodularisation remains low [11].

This paper presents a novel development of software remodularization applied to the contemporary challenge of discovering fine-grained MSs from an ES's code. It extends the syntactic focus of software remodularization, by exploiting the semantic structure of ESs, i.e., BOs and their relationships, which are, in principle, influential in class cohesion and coupling. Specifically, the paper presents the following:

- A novel MS discovery method for ESs combining syntactic properties, derived from extracted structural inheritance relationships, structural interaction relationships, structural class similarity, and semantic properties, derived in turn from databases and the relationships of BOs managed by classes. 
- An evaluation of the MS discovery methods that addresses three research questions (refer Sect.4.1) by implementing a prototype and experimenting on two open-source CRMs: SugarCRMonote ${ }^{1}$ and ChurchCRM ${ }^{2}$. The results show that there is a $26.46 \%$ and $2.29 \%$ improvement in cohesion and a $18.75 \%$ and $16.74 \%$ reduction in coupling between modules of SugarCRM and ChurchCRM, respectively. Furthermore, SugarCRM and ChurchCRM manage to achieve $3.7 \%$ and $31.6 \%$ improved system execution efficiency and $36.2 \%$ and $47.8 \%$ scalability improvement, respectively, when MSs are introduced to the system as suggested by our approach while preserving overall system availability (refer to Tables 1, 2, 3, 4, 5 and 6).

The remainder of the paper is structured as follows. Section 2 describes the related works and background on system remodularization techniques. Section 3 provides a detailed description of our MS discovery approach while Sect. 4 describes the implementation and evaluation. The paper concludes with Sect. 5 .

\section{Background and Motivation}

This section first provides an overview of existing software remodularization and MS discovery techniques with their relative strengths and weaknesses. It then provides an overview of the architectural context of ESs and their alignments with MSs. This context is assumed in the presentation of our software remodularization techniques (Sect.3).

\subsection{Related Work and Techniques Used for Software Remodularization}

Software remodularization techniques involve automated analysis of different facets of systems, including software structure, behaviour, functional requirements, and non-functional requirements. Techniques have focussed on static analysis to analyse code structure and database schemas of the software systems while dynamic analysis studies interactions of systems. Both approaches provide complementary information for assessing properties of system modules based on high cohesion and low coupling, and make recommendations for improved modularity. However, static analysis is preferable for broader units of analysis (i.e., systems or subsystems level) as all cases of systems' execution are covered compared to dynamic analysis [9].

Traditionally, research into software remodularization based on static analysis has focused on a system's implementation through two areas of coupling and cohesion evaluation. The first is structural coupling and cohesion, which focuses on structural relationships between classes in the same module or in different modules. These include structural inheritance relationships between classes and structural interaction relationships resulting when one class creates another class

\footnotetext{
${ }^{1}$ https://www.sugarcrm.com/.

${ }^{2}$ http://churchcrm.io/.
} 
and uses an object reference to invoke its methods [8]. Structural relationships such as these are automatically profiled through Module Dependency Graphs (MDG), capturing classes as nodes and structural relationships as edges [8,9], and are used to cluster classes using K-means, Hill-climbing, NSGA II and other clustering algorithms. The second is structural class similarity (otherwise known as conceptual similarity of the classes) [10]. This draws from information retrieval (IR) techniques, for source code comparison of classes, under the assumption that similarly named variables, methods, object references, tables and attributes in database query statements, etc., infer conceptual similarity of classes. Relevant terms are extracted from the classes and used for latent semantic indexing and cosine comparison to calculate the similarity value between them. Class similarity, thus, provides intra-module measurements for evaluating coupling and cohesion, in contrast to the inter-module measurements applied through structural coupling and cohesion described above.

Despite many proposals for automated analysis of systems, studies show that the success rate of software remodularization remains low [11]. A prevailing problem is the limited insights available from purely syntactic structures of software code to derive structural and interactional relationships of modules. More recently, semantic insights available through BO relationships were exploited to improve the feasibility of architectural analysis of applications. ESs manage domain-specific information using BOs, through their databases and business processes [5]. Evaluating the BO relationships and deriving valuable insights from them to remodularize software systems falls under the category of semantic structural relationships analysis. Such semantic relationships are highlighted by the experiments conducted by Pẽrez-Castillo et al. [12], in which the transitive closure of strong BO dependencies derived from databases was used to recommend software function hierarchies, and by the experiments conducted by $\mathrm{Lu}$ et al. [13], in which SAP ERP logs were used to demonstrate process discovery based on BOs. Research conducted by De Alwis et al. $[14,15]$ on MS discovery based on BO relationship evaluation shows the impact of considering semantic structural relationships in software remodularization. However, to date, techniques related to semantic structural relationships have not been integrated with syntactic structural relationships and structural class similarity techniques. As a result, currently proposed design recommendation tools provide insufficient insights for software remodularization.

\subsection{Architecture for Enterprise System to Microservice Remodularization}

As detailed in Sect. 2.1, there are multiple factors which should be considered in the MS derivation process. In this section, we define the importance of considering such factors with respect to the architectural configuration of the ES and MSs.

As depicted in Fig. 1, an ES consists of a set of self-contained modules drawn from different subsystems and is deployed on a "backend" platform. Modules consist of a set of software classes which contain internal system 


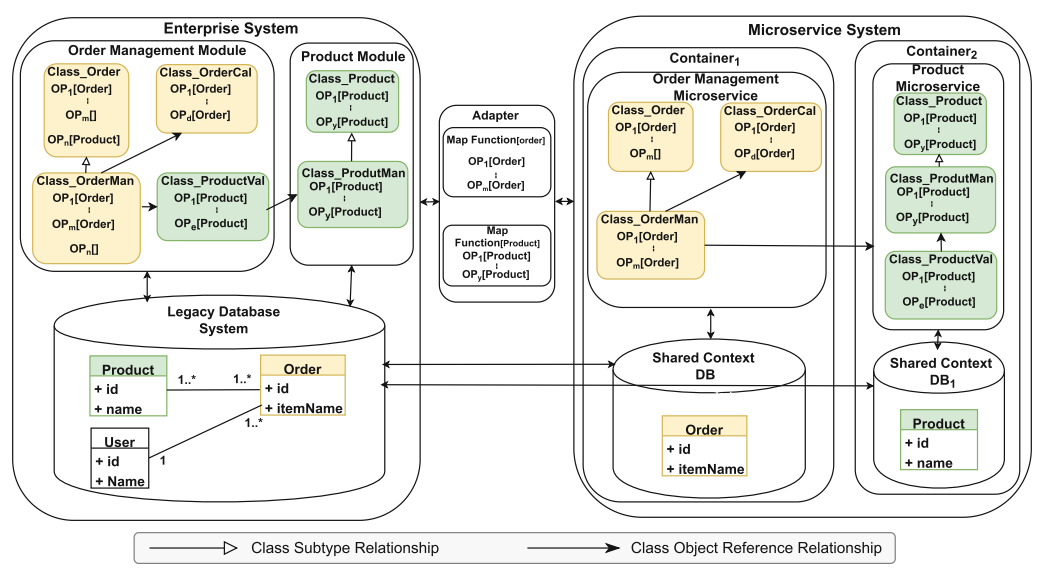

Fig. 1. Overview of an enterprise system extended with extracted microservices.

operations and operations which manage one or more BOs through create, read, update, and delete (CRUD) operations. For example 'Order Management Module' consists of several classes such as 'Class_Order', 'Class_OrderCal' and 'Class_OrderMan', which contain operations manipulating data related to 'Order' BO and 'Class_ProductVal', which contain operations manipulating data related to 'Product' BO. Furthermore, the modules are interrelated through method calls between classes in different modules (see the relationship of 'Class_ProductVal' and 'Class_ProductMan' in Fig. 1). In addition, classes inside each individual module can have generalization/specialization relationships (i.e., subtype-supertype relationships) between different classes as depicted by the relationships between 'Class_Order' and 'Class_OrderMan', and 'Class_Product' and 'Class_ProductMan' in Fig. 1.

The MSs, on the other hand, support a subset of operations through classes which are related to individual BOs. Such implementations lead to high cohesion within MSs and low coupling between the MSs (see the 'Order Management Microservice' and 'Product Microservice' in Fig. 1). The MSs communicate with each other through API calls in case they require information related to different BOs which reside in other MSs. For example, 'Order Management Microservice' can acquire Product values through an API call to 'Product Microservice' (refer arrow between the MSs in Fig. 1). The execution of operations across the ES and MS system is coordinated through business processes, which means that invocations of BO operations on the MSs will trigger operations on ES functions involving the same BOs. As required for consistency in an MS system, BO data will be synchronised across databases managed by ES and MSs periodically.

Based on this understanding of the structure of the ES and MSs, it is clear why we should consider semantic and syntactic information for the MS discovery process. In order to capture the subtype relationships and object reference relationships that exist in the ES system, we need structural inheritance relationship 
and structural interaction relationship analysis methods. Such methods can help to group classes which are highly coupled into one group, such as the grouping of 'Class_Order', 'Class_OrderCal' and 'Class_OrderMan' into one 'Order Management Microservice', as depicted in Fig. 1. However, those relationships alone would not help to capture class similarities at the code level. For example, the 'Class_ProductVal' operates on 'Product' BO and relates to the 'Product Module' much more than the 'Order Management Module'. Such information can be captured using structural class similarity measuring methods. With structural inheritance and interaction relationships and structural class similarity we can cluster classes into different modules. However, such modules might not align with the domain they are related to until we consider the BO relationships of different classes. In Fig. 1, one can notice that different classes in the ES relate to different BOs. As such, it is of utmost importance to consider the semantic structural relationships in the MS derivation process, since each MS should aim to contain classes that are related to each other and perform operations on the same BO (refer to the 'Order Management Microservice' and 'Product Microservice' in Fig. 1).

Previous research has extensively used structural relationships in system remodularization [8-10]. However, when it comes to MS derivation, combining the semantic structural relationships with the syntactic structural relationships should allow deriving better class clusters suitable for MS implementation. Given this system architecture context and our understanding of the features that should be evaluated for MS systems, we developed algorithms, as described in Sect.3, for MS discovery. We use the following formalisation here onwards to describe the algorithms.

Let $\mathbb{I}, \mathbb{O}, \mathbb{O P}, \mathbb{B}, \mathbb{T}$ and $\mathbb{A}$ be a universe of input types, output types, operations, BOs, database tables and attributes respectively. We characterize a database table $t \in \mathbb{T}$ by a collection of attributes, i.e., $t \subseteq \mathbb{A}$, while a business object $b \in \mathbb{B}$ is defined as a collection of database tables, i.e., $b \subseteq \mathbb{T}$. An operation op, either of an ES or MS system, is given as a triple $(I, O, T)$, where $I \in \mathbb{I}^{*}$ is a sequence of input types the operation expects for input, $O \in \mathbb{O}^{*}$ is a sequence of output types the operation produces as output, and $T \subseteq \mathbb{T}$ is a set of database tables the operation accesses, i.e., either reads or augments. ${ }^{3}$ Each class cls $\in C L S$ is defined as a collection of operations, i.e., cls $\subseteq \mathbb{O P}$.

\section{Clustering Recommendation for Microservice Discovery}

In order to derive the MSs while considering the factors defined in Sect.2, we developed a six-step approach, which is illustrated in Fig. 2. In the first step, we derive the BOs by evaluating the SQL queries in the source code structure and also the database schemas and data as described by Nooijen et al. [16]. Next, we analyse the semantic structural relationships by deriving the class and $\mathrm{BO}$

\footnotetext{
${ }^{3} A^{*}$ denotes the application of the Kleene star operation to set $A$.
} 
relationships. Steps 3-5 are used to discover the syntactic details related to the ES. In the third step, we measure the structural class similarities between the classes and in steps 4 and 5 we capture the structural details of the classes, step 4 discovers the structural inheritance relationships and step 5 discovers the structural interaction relationships. The details obtained through steps $2-5$ are provided to the final step in which a K-means clustering algorithm is used to cluster and evaluate the best possible combination of classes for MS development and finally suggest them to the developers. Detailed descriptions of these steps and corresponding algorithms are provided in Sect.3.1.

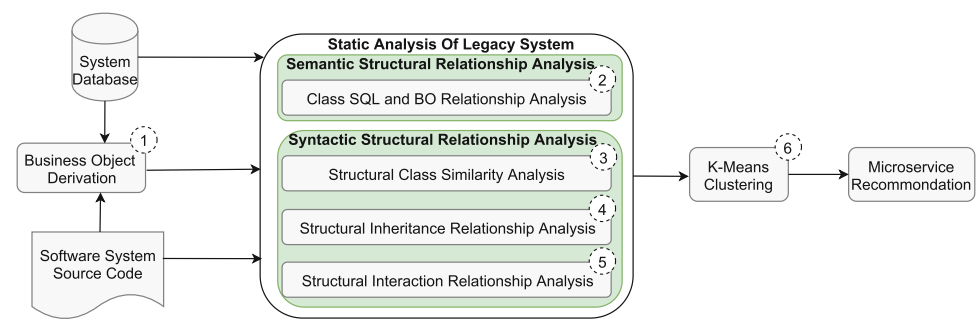

Fig. 2. Overview of our microservice discovery approach.

\subsection{Clustering Discovery Algorithms}

As depicted in Fig. 2, in order to derive a satisfactory clustering of system classes and operations and suggest MSs recommendations, we supply the K-means algorithm with four main feature sets. To derive these feature sets, we use Algorithm 1 , which is composed of eight steps.

We define a BO $b \in B$ in an ES as a collection of database tables, i.e., $b \subseteq T$, since information related to a $\mathrm{BO}$ is often stored in several database tables. The $B O S$ function in Algorithm 1 used to derive BOs $B$ from ESs as detailed by Nooijen et al. [16] (see line 1). In the second step of the algorithm, the function $C L S E X T$ is used to extract code related to each class $c l s \in C L S$ from the system code by searching through its folder and package structure (see line 2).

In the third step, we extract information required for the structural class similarity analysis using information retrieval (IR) techniques. As such, in the third step, the algorithm identifies unique words $U W$ related to all the classes using function $U W O R D E X T$ (see line 3) which requires all the source codes of the classes $C L S$, and stop words $S T W$, which should be filtered out from the classes. In general, IR techniques analyse documents and filter out the content which does not provide any valuable information for document analysis, which are referred to as 'stop words'. In our case, the stop words $(S T W)$ contain syntax related to the classes, common technical terms used in coding in that particular language (in this case PHP) and also common English words which would not provide any valuable insight about class purpose. These are specified by the user based on the language of the system they evaluate. The function UWORDEXT 
first filters out the stop words $S T W$ from the classes $C L S$ and then identifies the collection of unique words $U W$ in classes $C L S$, which is generally referred to as a 'bag of words' [17].

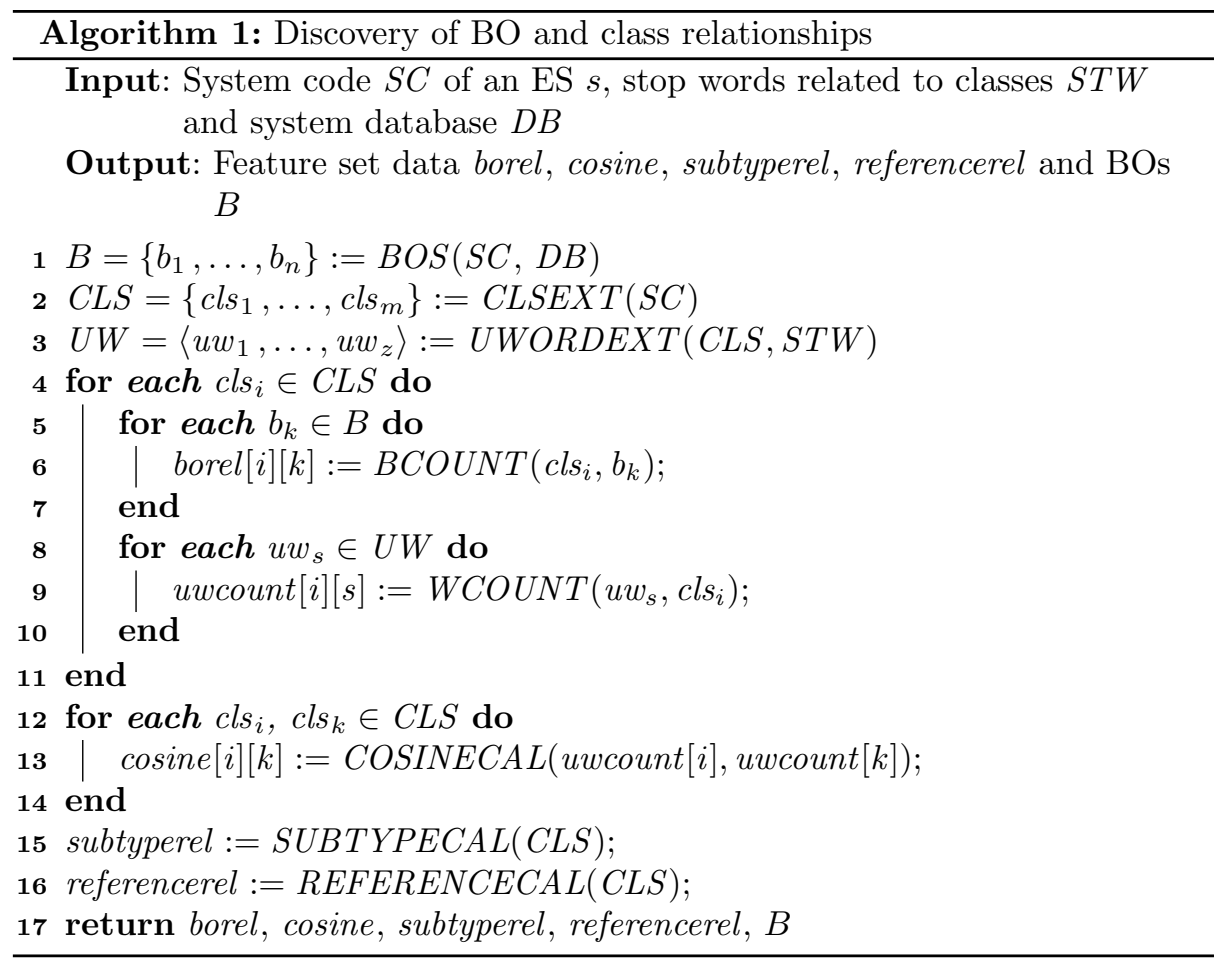

In the fourth step, the algorithm evaluates each class ( cls $\in C L S$ ) extracted in step two and identifies the BOs which are related to each class. For this purpose, the algorithm uses the function BCOUNT that processes the SQL statements, comments and method names related to the classes and counts the number of times tables relate to BOs. This information is stored in matrix borel (see lines 5-7). In this matrix, each row represents a class, and each column represents the number of relationships that class has with the corresponding BO, as depicted in Fig. 3(a). This helps to capture the semantic structural relationships (i.e., BO relationships) data, which provides an idea about the "boundness" of classes to BOs. For example Class 1 'Cls 1' is related to 'BO1' and 'BO2' in Fig. 3(a).

In the fifth step, the algorithm derives another matrix uwcount, which keeps the count of unique words related to each class using the function WCOUNT (see lines 8-10). In this matrix, again, rows correspond to classes, and columns correspond to unique words identified in step three of the algorithm that appear in the corresponding classes. The values in uwcount are then used in the sixth step to calculate the cosine similarity between the documents using COSINECAL function (see lines 12-14). First, this function normalizes the term frequencies 
with the respective magnitude L2 norms. Then it calculates the cosine similarity between different documents, by calculating the cosine value between two vectors of the uwcount (i.e., the rows related to two classes in uwcount matrix) and stores the values in the cosine matrix, as exemplified in Fig. 3(b). Note that the cosine similarity of a class to itself is always ' 1 '. This provides the structural class similarity data for clustering.

Next, we extract the structural inheritance relationships (i.e., the class subtype relationships) and structural interaction relationships (i.e., the class object reference relationships). This is achieved through steps seven and eight in the algorithm which use function SUBTYPECAL (see line 15) to identify the subtype relationships and function REFERENCECAL (see line 16) to identify the class object reference relationships. In both of these functions, as the first step, the code is evaluated using Mondrian ${ }^{4}$, which generates graphs based on class relationships. Then, the graphs are analyzed to create two matrices, namely subtyperel and referencerel which, respectively summarize the class subtype and reference relationships for further processing (see subtyperel depicted in Fig. 3(c) and referencerel depicted in Fig. 3(d)).

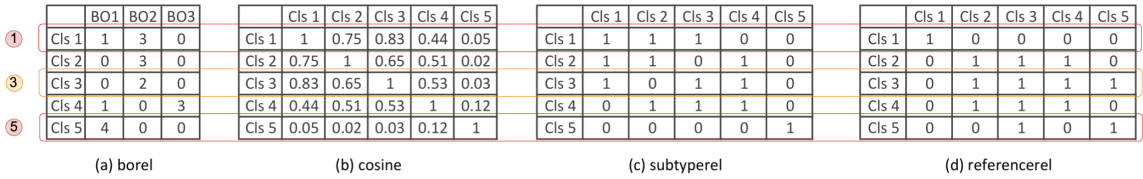

Fig. 3. Matrices derived from Algorithm 1.

The feature set data borel, cosine, subtyperel, referencerel and BOs $B$ obtained from Algorithm 1 are provided as input to the K-Means algorithm (i.e., Algorithm 2) to cluster the classes related to BOs based on their syntactic and semantic relationships. Note that each dataset captures different aspects of relationships between classes in the given system (see Fig. 3). Each initial centroid intcent $\in$ IntCent is a row number in the dataset that we provide. For example, one can select the first row of the dataset (as we have done in Fig. 3, see highlighted in red), as an initial centroid. In that situation, the IntCent will contain the data related to that specific row of the data set. Given these datasets as the first step in Algorithm 2, we initialize the distance difference value distDif to some constant, e.g., 10. The distDif is responsible for capturing the distance differences between the initial centroids IntCent and the newly calculated centroids NewCent. If this distance difference is zero, then it means that there is no difference between the initial centroid values and the newly calculated centroid values (in which case the algorithm terminates). After initializing the distDif value, the

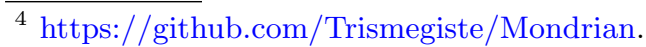


next steps of the algorithm are performed iteratively until the aforementioned condition of distDif is met (see lines 2-21).

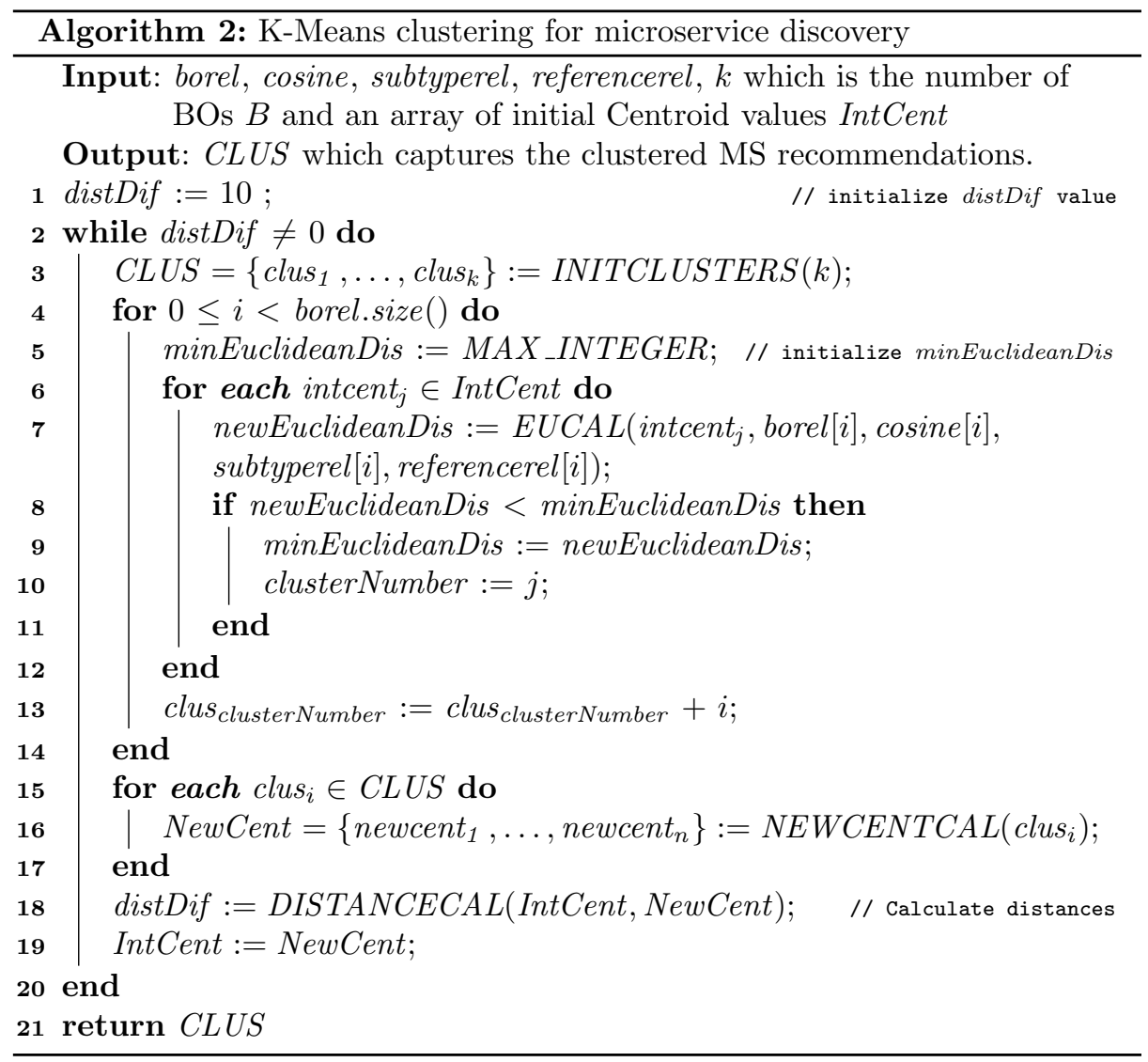

The first step of the iterative execution is to initialize the set of clusters $C L U S$, which we use to store the node groups identified by Algorithm 2. Next, we need to identify the cluster that each row (or the node) of our data should belong to by comparing the distance between each node in the dataset and each node in the initial centroids intcent $\in$ IntCent. Hence we iterate through each row of the dataset we obtained from Algorithm 1 (see line 4 in Algorithm 2), while calculating the Euclidean distance between each row and each initial centroid intcent $\in$ IntCent (see lines 4-12 in Algorithm 2). For this calculation, as the initial step, we define the minimum Euclidean distance value minEquclidianDis and initialize it to MAX_INTEGER (e.g., 100000). We assign this value to the minEquclidianDis to ensure that it would be larger than the value we obtain for the newEuclideanDis (line 7) at the end of the first iteration. Then, we calculate the Euclidean distance between one data set, for example, row 1 in Fig. 3 and each initial centroid point given. Next, we identify the centroid which has the 
minimum Euclidian distance to the node we obtained and allocate that node number to that particular cluster clus $\in$ CLUS (line 13). This process is carried out until all the nodes are clustered based on the Euclidean distance calculation. In the end, each node in the data set is clustered towards the centroid which has the minimal distance to it based on the four feature sets which emphasize that the classes related to that particular cluster are bound to the same $\mathrm{BO}$ and to each other syntactically and semantically.

The next step of Algorithm 2 is to calculate the new centroids based on the clusters obtained. For this, we take the mean value of the node data sets belonging to each cluster and assign it as the new centroid (see function NEWCENTCAL at lines 15-17 in Algorithm 2). Then, we calculate the distance difference between the initial centroids and the new centroids. If this difference is zero, it means that there is no change of the centroid points and the algorithm has come to the optimum clustering point. If not, the newly calculated centroids becomes the initial centroids for the next iteration of the algorithm. At the end of the algorithm, the final set of clusters which contain the classes of the analysed ES are provided to the developers as recommendations for constructing MSs based on them.

\section{Implementation and Validation}

To demonstrate the applicability of the method described in Sect. 3, we developed a prototypical MS recommendation system ${ }^{5}$ capable of discovering the class clusters related to different BOs, which lead to different MS configurations. The system was tested against two open-source customer relationship management systems: SugarCRM and ChurchCRM. SugarCRM consists of more than 8,000 files and 600 attributes in 101 tables, while ChurchCRM consists of more than 4,000 files and 350 attributes in 55 tables. However, most of the files are HTML files which are related to third-party components used by the systems. For the clustering, we only used the 1,400 classes of SugarCRM and 280 classes of ChurchCRM which capture the core functionality of the systems. Using our implementation, we performed static analysis of the source code to identify the BOs managed by the systems. As a result, 18 BOs were identified in SugarCRM, e.g., account, campaign, and user, and 11 BOs in ChurchCRM, e.g., user, family, and email. Then, we performed static analysis of both systems to derive matrices, similar to those depicted in Fig. 3, summarizing the BO relationships, class similarity relationships, class subtype relationships and class object reference relationships. All the obtained results were processed by the prototype to identify the class clusters to recommend MSs. Based on the input, the prototype identified 18 class clusters related to the BOs in SugarCRM and 11 class clusters related to the BOs in ChurchCRM. Consequently, each cluster suggests classes for developing an MS that relates to a single BO.

\footnotetext{
${ }^{5}$ https://github.com/AnuruddhaDeAlwis/KMeans.git.
} 


\subsection{Research Questions}

Our evaluation aims to answer three research questions:

- RQ1: Do syntactic and semantic relationships between source code artifacts of an ES convey useful information for its remodularization into MSs?

- RQ2: Can our MS recommendation system discover MSs that have better cohesion and coupling than the original ES modules and lead to high scalability, availability, and execution efficiency in the cloud environment?

- RQ3: Can our MS recommendation system discover MSs that lead to better scalability, availability, and execution efficiency in the cloud environment than some MSs that do not follow the recommendations?

\subsection{Experimental Setup}

To answer the above research questions, we set up the following experiment consisting of three steps. In the first step, we evaluated the effectiveness of considering four different features (i.e., feature 1: borel, feature 2: cosine, feature 3: referencerel and feature 4: subtyperel extracted in Algorithm 1) in the clustering process. We evaluated this by measuring the Lack of Cohesion (LOC) and Structural Coupling (StrC) of the clusters, as detailed by Candela et al. [11], while incrementally adding different features in the clustering process. The values for the ES was calculated by clustering the classes into folders while conserving the original package structure, see first rows in Tables 1, 2, 3 and 4 . Then, we clustered the classes several times, each time adding more features and calculating the LOC and StrC values. The obtained values are reported in Tables 1, 2, 3 and 4 .

After evaluating the effectiveness of various features for clustering, we assessed the efficacy of introducing MSs to the ES. To this end, first, we hosted each ES in an AWS cloud by creating two EC2 instances having two virtual CPUs and a total memory of $2 \mathrm{~GB}$, as depicted on the left side of Fig. 4. Systems' data were stored in a MySQL relational database instance which has one virtual CPU and total storage of $20 \mathrm{~GB}$. Afterward, these systems were tested against 100 and 200 executions generated by four machines simultaneously, simulating the customer requests. We recorded the total execution time, average CPU consumption, and average network bandwidth consumption for these executions (refer to our technical report [18]). For SugarCRM, we test the functionality related to campaign creation, while for ChurchCRM we test the functionality related to adding new people to the system. The simulations were conducted using Selenuim ${ }^{6}$ scripts which ran the system in a way similar to a real user.

Next, we introduced the 'campaign' and 'user' MSs to the SugarCRM system and 'person' and 'family' MSs to the ChurchCRM system. As depicted on the right side of Fig. 4, we hosted each MS on an AWS elastic container service (ECS), containing two virtual CPUs and a total memory of $1 \mathrm{~GB}$. The BO data of each MS (i.e., campaign BO and user BO data of SugarCRM and person BO

\footnotetext{
${ }^{6}$ https://www.seleniumhq.org/.
} 
and family BO data of ChurchCRM) was stored in separate MySQL relational database instances with one virtual CPU and total storage of $20 \mathrm{~GB}$. Afterward, the test were performed on both ESs, again simulating campaign creation for SugarCRM and adding new people for ChurchCRM. Since MSs are refactored parts of the ESs in these tests, the ESs used API calls to pass the data to the MSs and the MSs processed and sent back the data to the ESs. MS databases and the ES databases were synchronized using the Amazon database migration service. Again, we recorded the total execution time, average CPU consumption, and average network bandwidth consumption for the entire system (i.e., ES and MS as a whole) (refer to our technical report [18]). The scalability, availability and execution efficiency of the systems were calculated based on the attained values. The results obtained are summarized in Tables 5 and 6 as ES with MSs(1) (refer to the second rows in Tables 5 and 6). Scalability was calculated according to the resource usage over time, as described by Tsai et al. [19]. To determine availability, first we calculated the packet loss for one minute when the system is down and then obtained the difference between the total up time and total time (i.e., up time + down time), as described by Bauer et al. [20]. Dividing the total time taken by the legacy system to process all requests by the total time taken by the corresponding ES system which has MSs led to the calculation of efficiency gain.

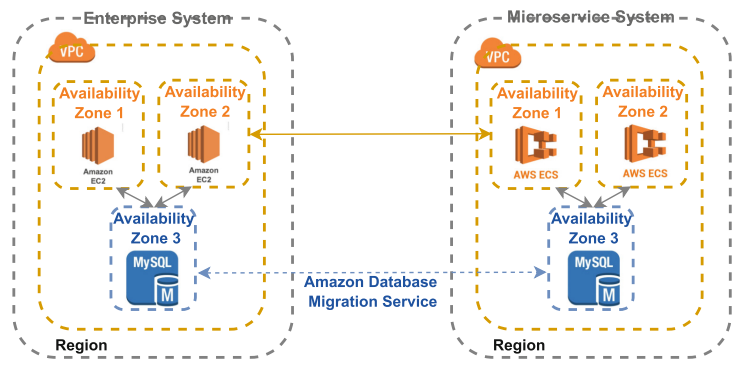

Fig. 4. System implementation in AWS.

In the third experiment, we disrupted the suggestions provided by our recommendation system and developed 'campaign' and 'user' MSs for SugarCRM, while introducing operations related to 'campaign' to 'user' MS and operations related to 'user' to 'campaign' MS. Similarly, for ChurchCRM, we developed 'person' and 'family' MSs such that 'person' MS contains operations related to 'family' MS and 'family' MS contains operations related to 'person' MS. With this change, again, we set up the experiment as described earlier and obtained the experimental results (refer to our technical report [18]). Then we calculated the scalability, availability and execution efficiencies of the systems which are summarized in Tables 5 and 6 as ES with $M S s$ (2) (refer to the third rows in Tables 5 and 6). Based on these obtained experimental results we evaluate the effectiveness of the algorithms by answering the posed research questions. 
RQ1: Impact of Syntactic and Semantic Relationships. The lower the lack of cohesion and structural coupling numbers, the better the cohesion and coupling of the system [11]. Consequently, it is evident from the average numbers reported in Tables 1, 2, 3 and 4 (refer to the orange color cells) that clustering improved the cohesion of software modules of SugarCRM and ChurchCRM by $26.46 \%$ and $2.29 \%$, respectively, while reducing the coupling between modules by $18.75 \%$ and $16.74 \%$ respectively. Furthermore, it is evident that introducing additional features (i.e., syntactic and semantic information) in the clustering process increased the number of modules which obtain better coupling and cohesion values (refer to the blue cells in Tables 1, 2, 3 and 4). Thus, we conclude that there is a positive effect of introducing multiple syntactic and semantic relationships to the clustering process to improve the overall performance of the system.

RQ2: Recommended MSs vs Original ES. According to Tsai et al. [19], the lower the measured number, the better the scalability. Thus, it is evident that the MS systems derived based on our clustering algorithm managed to achieve $3.7 \%$ and $31.6 \%$ improved system execution efficiency and $36.2 \%$ and $47.8 \%$ scalability improvement (considering CPU scalability) (refer Tables 5 and 6), for SugarCRM and ChurchCRM, respectively, while also achieving better cohesion

Table 1. ChurchCRM ES vs MS system lack of cohesion value comparison.

\begin{tabular}{l|c|c|c|c|c|c|c|c|c|c|c|c}
\hline Features & 1 & 2 & 3 & 4 & 5 & 6 & 7 & 8 & 9 & 10 & 11 & Avg \\
\hline \hline Original ES & 61 & 188 & 853 & 7 & 4 & 1065 & 31 & 378 & 3064 & 13 & 17 & 516.45 \\
\hline 1 and 2 & 61 & 77 & 666 & 33 & 8 & 1453 & 73 & 351 & 3802 & 3 & 10 & 594.27 \\
\hline 1,2 and 3 & 61 & 77 & 853 & 3 & 4 & 1564 & 23 & 351 & 3064 & 13 & 17 & 548.18 \\
\hline $1,2,3$ and 4 & 58 & 188 & 820 & 7 & 3 & 1059 & 31 & 351 & 3012 & 10 & 15 & 504.90 \\
\hline
\end{tabular}

Table 2. ChurchCRM ES vs MS system structural coupling value comparison.

\begin{tabular}{l|c|c|c|c|c|c|c|c|c|c|c|c}
\hline Features & 1 & 2 & 3 & 4 & 5 & 6 & 7 & 8 & 9 & 10 & 11 & Avg \\
\hline \hline Original ES & 41 & 26 & 61 & 17 & 16 & 70 & 29 & 31 & 123 & 27 & 19 & 41.81 \\
\hline 1 and 2 & 41 & 25 & 8 & 37 & 20 & 64 & 33 & 31 & 121 & 3 & 7 & 35.45 \\
\hline 1,2 and 3 & 41 & 25 & 61 & 16 & 16 & 68 & 29 & 27 & 123 & 7 & 19 & 41.09 \\
\hline $1,2,3$ and 4 & 42 & 25 & 34 & 17 & 15 & 63 & 29 & 3 & 112 & 26 & 17 & 34.81 \\
\hline
\end{tabular}

Table 3. SugarCRM ES vs MS system lack of cohesion value comparison.

\begin{tabular}{l|c|c|c|c|c|c|c|c|c|c|c|c|c|c|c|c|c|c|c}
\hline Features & 1 & 2 & 3 & 4 & 5 & 6 & 7 & 8 & 9 & 10 & 11 & 12 & 13 & 14 & 15 & 16 & 17 & 18 & Avg \\
\hline \hline Original ES & 32 & 19 & 1255 & 698 & 1482 & 0 & 163 & 693 & 349 & 45 & 171 & 1803 & 1058 & 0 & 66 & 47 & 317 & 522 & 484.44 \\
\hline 1 and 2 & 19 & 0 & 1067 & 1122 & 1173 & 0 & 86 & 459 & 170 & 21 & 120 & 953 & 587 & 0 & 36 & 6 & 453 & 187 & 358.83 \\
\hline 1,2 and 3 & 19 & 0 & 1201 & 626 & 1173 & 0 & 86 & 459 & 170 & 45 & 120 & 1027 & 587 & 0 & 36 & 7 & 590 & 268 & 356.33 \\
\hline $1,2,3$ and 4 & 19 & 0 & 1201 & 626 & 1173 & 0 & 86 & 459 & 170 & 45 & 120 & 1027 & 587 & 0 & 36 & 5 & 590 & 268 & 356.22 \\
\hline
\end{tabular}


Table 4. SugarCRM ES vs MS system structural coupling value comparison.

\begin{tabular}{l|c|c|c|c|c|c|c|c|c|c|c|c|c|c|c|c|c|c|c}
\hline $\begin{array}{l}\text { Features } \\
\text { considered }\end{array}$ & 1 & 2 & 3 & 4 & 5 & 6 & 7 & 8 & 9 & 10 & 11 & 12 & 13 & 14 & 15 & 16 & 17 & 18 & Avg \\
\hline \hline Original ES & 24 & 12 & 121 & 63 & 48 & 4 & 99 & 24 & 29 & 29 & 28 & 101 & 67 & 0 & 16 & 10 & 82 & 85 & 46.77 \\
\hline 1 and 2 & 12 & 2 & 117 & 76 & 48 & 4 & 87 & 18 & 25 & 20 & 27 & 85 & 45 & 0 & 15 & 5 & 52 & 64 & 39.00 \\
\hline 1,2 and 3 & 12 & 2 & 116 & 53 & 48 & 4 & 87 & 18 & 22 & 29 & 27 & 79 & 45 & 0 & 15 & 5 & 41 & 83 & 38.11 \\
\hline $1,2,3$ and 4 & 12 & 2 & 116 & 53 & 48 & 4 & 87 & 18 & 22 & 29 & 27 & 79 & 45 & 0 & 15 & 3 & 41 & 83 & 38.00 \\
\hline
\end{tabular}

Table 5. Legacy vs MS system EC2 characteristics comparison for SugarCRM.

\begin{tabular}{l|l|l|l|l|l|l|l}
\hline System type & $\begin{array}{l}\text { Scalability } \\
\text { [CPU] }\end{array}$ & $\begin{array}{l}\text { Scalability } \\
\text { [DB CPU] }\end{array}$ & $\begin{array}{l}\text { Scalability } \\
\text { network }\end{array}$ & $\begin{array}{l}\text { Availability } \\
{[200]}\end{array}$ & $\begin{array}{l}\text { Availability } \\
{[400]}\end{array}$ & $\begin{array}{l}\text { Efficiency } \\
{[200]}\end{array}$ & $\begin{array}{l}\text { Efficiency } \\
{[400]}\end{array}$ \\
\hline ES only & 3.521 & 2.972 & 2.759 & 99.115 & 99.087 & 1.000 & 1.000 \\
ES with MSs $(1)$ & 2.246 & 2.532 & 2.352 & 99.082 & 99.086 & 1.037 & 1.000 \\
ES with MSs $(2)$ & 2.667 & 2.546 & 2.684 & 99.099 & 99.099 & 1.018 & 0.986 \\
\hline
\end{tabular}

Table 6. Legacy vs MS system EC2 characteristics comparison for ChurchCRM.

\begin{tabular}{l|l|l|l|l|l|l|l}
\hline System type & $\begin{array}{l}\text { Scalability } \\
\text { [CPU] }\end{array}$ & $\begin{array}{l}\text { Scalability } \\
\text { [DB CPU] }\end{array}$ & $\begin{array}{l}\text { Scalability } \\
\text { network }\end{array}$ & $\begin{array}{l}\text { Availability } \\
{[200]}\end{array}$ & $\begin{array}{l}\text { Availability } \\
{[400]}\end{array}$ & $\begin{array}{l}\text { Efficiency } \\
{[200]}\end{array}$ & $\begin{array}{l}\text { Efficiency } \\
{[400]}\end{array}$ \\
\hline ES only & 3.565 & 3.109 & 3.405 & 99.385 & 99.418 & 1.000 & 1.000 \\
ES with MSs $(1)$ & 1.859 & 2.751 & 3.663 & 95.000 & 94.871 & 1.316 & 1.189 \\
ES with MSs(2) & 2.876 & 2.667 & 2.779 & 95.238 & 95.000 & 1.250 & 1.158 \\
\hline
\end{tabular}

and coupling values (refer Tables 1, 2, 3 and 4). As such, our recommendation system discovers MSs that have better cohesion and coupling values than the original enterprise system modules and can achieve improved cloud capabilities such as high scalability, high availability and high execution efficiency.

RQ3: Recommended MSs vs Some MSs. MSs developed based on the suggestions provided by our recommendation system for SugarCRM and ChurchCRM managed to achieve: (i) $36.2 \%$ and $47.8 \%$ scalability improvement in EC2 instance CPU utilization, respectively; (ii) $14.8 \%$ and $11.5 \%$ scalability improvement in database instance CPU utilization, respectively; (iii) while achieving $3.7 \%$ and $31.6 \%$ improvement in execution efficiency, respectively. However, MSs that violate the recommendations reduced (i) EC2 instance CPU utilization to $24.24 \%$ and $19.32 \%$; (ii) execution efficiency to $1.8 \%$ and $2.5 \%$, for SugarCRM and ChurchCRM respectively and reduced database instance CPU utilization to $14.3 \%$ for SugarCRM. As such, it is evident that the MSs developed by following the recommendations of our system provided better cloud characteristics than the MSs developed against these recommendations.

\subsection{Limitations}

Next, we discuss an important limitation of our approach.

Limitation of Structural Class Similarity Analysis: The cosine values might not provide an accurate idea about the structural class similarity since the structural similarity may also depend on the terms used in the definitions of 
the class names, method names and descriptions given in comments. This was mitigated to a certain extent by evaluating the code structure of the software systems before evaluating and verifying that the class names, method names and comments provide valuable insights into the logic behind the classes that implement the system.

\section{Conclusion}

This paper presented a novel technique for automated analysis and remodularization of ESs as MSs by combining techniques which consider semantic knowledge, together with syntactic knowledge about the code of the systems. A prototype recommendation system was developed and validation was conducted by implementing the MSs recommended by the prototype for two open source ESs: SugarCRM and ChurchCRM. The experiment showed that the proposed technique managed to derive class clusters which would lead to MSs with desired Cloud characteristics, such as high cohesion, low coupling, high scalability, high availability, and processing efficiency. In future work, we will enhance the technique by considering method level relationships in the analysis of MS candidates.

Acknowledgment. This work was supported in part, through the Australian Research Council Discovery Project: DP190100314, "Re-Engineering Enterprise Systems for Microservices in the Cloud".

\section{References}

1. Newman, S.: Building Microservices. O'Reilly Media Inc., Sebastopol (2015)

2. https://www.nginx.com/blog/microservices-at-netflix-architectural-bestpractices/

3. Barros, A., Duddy, K., Lawley, M., Milosevic, Z., Raymond, K., Wood, A.: Processes, roles, and events: UML concepts for enterprise architecture. In: Evans, A., Kent, S., Selic, B. (eds.) UML 2000. LNCS, vol. 1939, pp. 62-77. Springer, Heidelberg (2000). https://doi.org/10.1007/3-540-40011-7_5

4. Schneider, T.: SAP Business ByDesign Studio: Application Development, pp. 2428. Galileo Press, Boston (2012)

5. Decker, G., Barros, A., Kraft, F.M., Lohmann, N.: Non-desynchronizable service choreographies. In: Bouguettaya, A., Krueger, I., Margaria, T. (eds.) ICSOC 2008. LNCS, vol. 5364, pp. 331-346. Springer, Heidelberg (2008). https://doi.org/10. 1007/978-3-540-89652-4_26

6. Barros, A., Decker, G., Dumas, M.: Multi-staged and multi-viewpoint service choreography modelling. In: Proceedings of the Workshop on Software Engineering Methods for Service Oriented Architecture (SEMSOA), Hannover, Germany. CEUR Workshop Proceedings, vol. 244, May 2007

7. Barros, A., Decker, G., Dumas, M., Weber, F.: Correlation patterns in serviceoriented architectures. In: Dwyer, M.B., Lopes, A. (eds.) FASE 2007. LNCS, vol. 4422, pp. 245-259. Springer, Heidelberg (2007). https://doi.org/10.1007/978-3540-71289-3_20 
8. Praditwong, K., Harman, M., Yao, X.: Software module clustering as a multiobjective search problem. IEEE Trans. Softw. Eng. 37(2), 264-282 (2010)

9. Mitchell, B.S., Mancoridis, S.: On the automatic modularization of software systems using the bunch tool. IEEE Trans. Softw. Eng. 32(3), 193-208 (2006)

10. Poshyvanyk, D., Marcus, A.: The conceptual coupling metrics for object-oriented systems. In: 22nd IEEE International Conference on Software Maintenance, pp. 469-478. IEEE, September 2006

11. Candela, I., Bavota, G., Russo, B., Oliveto, R.: Using cohesion and coupling for software remodularization: is it enough? ACM Trans. Softw. Eng. Methodol. (TOSEM) 25(3), 24 (2016)

12. Pérez-Castillo, R., García-Rodríguez de Guzmán, I., Caballero, I., Piattini, M.: Software modernization by recovering web services from legacy databases. J. Softw.: Evol. Process 25(5), 507-533 (2013)

13. Lu, X., Nagelkerke, M., van de Wiel, D., Fahland, D.: Discovering interacting artifacts from ERP systems. IEEE Trans. Serv. Comput. 8(6), 861-873 (2015)

14. De Alwis, A.A.C., Barros, A., Fidge, C., Polyvyanyy, A.: Business object centric microservices patterns. In: Panetto, H., Debruyne, C., Hepp, M., Lewis, D., Ardagna, C.A., Meersman, R. (eds.) OTM 2019. LNCS, vol. 11877, pp. 476-495. Springer, Cham (2019). https://doi.org/10.1007/978-3-030-33246-4_30

15. De Alwis, A.A.C., Barros, A., Polyvyanyy, A., Fidge, C.: Function-splitting heuristics for discovery of microservices in enterprise systems. In: Pahl, C., Vukovic, M., Yin, J., Yu, Q. (eds.) ICSOC 2018. LNCS, vol. 11236, pp. 37-53. Springer, Cham (2018). https://doi.org/10.1007/978-3-030-03596-9_3

16. Nooijen, E.H.J., van Dongen, B.F., Fahland, D.: Automatic discovery of datacentric and artifact-centric processes. In: La Rosa, M., Soffer, P. (eds.) BPM 2012. LNBIP, vol. 132, pp. 316-327. Springer, Heidelberg (2013). https://doi.org/10. 1007/978-3-642-36285-9_36

17. Lebanon, G., Mao, Y., Dillon, J.: The locally weighted bag of words framework for document representation. J. Mach. Learn. Res. 8(Oct), 2405-2441 (2007)

18. https://drive.google.com/file/d/19niZYleVsuboNETCScYRB9LFVi3_5F2z/view? usp $=$ sharing

19. Tsai, W.T., Huang, Y., Shao, Q.: Testing the scalability of SaaS applications. In: 2011 IEEE International Conference on Service-Oriented Computing and Applications (SOCA), pp. 1-4. IEEE, December 2011

20. Bauer, E., Adams, R.: Reliability and Availability of Cloud Computing. Wiley, Hoboken (2012) 